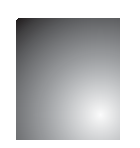

\title{
Gestão do Conhecimento e Vantagem Competitiva: ESTUDO NO SETOR METALMECÂNICO
}

\author{
Knowledge Management and Competitive Advantage: study in \\ the metal-mechanic sector
}

\section{Andressa Centenaro}

Mestranda em Administração. Faculdade Meridional. Passo Fundo, RS, Brasil. E-mail: centenaro.andressa@gmail.com

\section{Angela Maria Ortolan Bonemberger}

Mestranda em Administração. Faculdade Meridional. Passo Fundo, RS, Brasil. E-mail: angela.bonemberger@imed.edu.br

\section{Claudionor Guedes Laimer}

Doutor em Administração. Professor e pesquisador do Programa de Pós-Graduação em Administração da Faculdade Meridional. Passo Fundo, RS, Brasil. E-mail: laimer@imed.edu.br

\section{Resumo}

O estudo tem como objetivo analisar os fatores relacionados à cultura organizacional que auxiliam nos processos de gestão do conhecimento, colaborando para geração de vantagem competitiva nas empresas do setor metalmecânico. Para tanto, foi realizada uma pesquisa quantitativa com estratégia de levantamento de dados, mediante a aplicação de um questionário estruturado em uma amostra de 63 profissionais colaboradores de 13 empresas do setor metalmecânico. Os resultados foram analisados por meio da regressão múltipla $e$ indicam que para o segmento estudado, a aprendizagem e a confiança são preditores positivos e significativos da gestão do conhecimento. Dessa forma, pode-se inferir que as empresas que estimularem seus colaboradores para a aprendizagem e investirem na manutenção da confiança entre os membros da organização poderão obter melhor desempenho na gestão do conhecimento. Por fim, os resultados revelam, ainda, que a gestão do conhecimento influencia diretamente o desempenho $e$ a efetividade organizacional, demonstrando que o uso e o compartilhamento dos conhecimentos tácito e explícito são geradores de vantagem competitiva diante dos concorrentes.

Palavras-chaves: Vantagem Competitiva. Gestão do Conhecimento. Colaboração. Confiança. Aprendizagem.

\section{Abstract}

The study aims to analyze the factors related to organizational culture that assist in knowledge management processes, helping to generate competitive advantage in the companies of metal-mechanic sector. Therefore, a quantitative survey data collection strategy was performed by applying a structured questionnaire in a sample of 63 employees from 13 companies in the metal-mechanic sector. The results were analyzed using multiple regression and indicate that the studied segment, learning and trust are positive and significant predictors of knowledge management. Thus, it can be inferred that the companies that stimulate its employees to learn and invest in maintaining confidence among members of the organization may achieve better performance in knowledge management. Finally, the results also expose that the knowledge management directly influences the performance and organizational effectiveness, demonstrating that the use and sharing of tacit and explicit knowledge are generating competitive advantage to competitors.

Keywords: Competitive Advantage. Knowledge Management. Collaboration. Trust. Learning. 


\section{INTRODUÇÃo}

Na sociedade atual, o papel de gerar toda a riqueza e poder das organizações deixou de ser exclusividade dos fatores tradicionais de produção, como: terra, trabalho e capital. A partir da década de 1980, a literatura começa a explorar o papel dos recursos e das competencias internas das firmas como fonte de vantagem competitiva, sob a ótica da perspectiva teórica da visão baseada em recursos (WERNERFELT, 1984; DIERICKX; COOL, 1989; PRAHALAD; HAMEL, 1990; BARNEY, 1991). Os estudos realizados por esses autores diferenciavam-se da perspectiva baseada na Estrutura-Conduta-Desempenho, tida como convencional e que explicava a vantagem competitiva da organização pela sua capacidade de posicionar-se frente às forças do ambiente externo (PORTER, 1986). Muitos estudos na década de 1990 destacaram a manipulação dos recursos de conhecimento como crucial para as empresas competirem no mercado (GRANT, 1991; ZANDER; KOGUT, 1995).

Reconhecendo a importância de investir em conhecimento, as empresas passaram a buscar melhores formas de gerir e aplicar o conhecimento. Contudo, ainda é necessária a aplicação de modelos analíticos e estudos empíricos que explorem as implicações práticas dos processos envolvidos na criação do conhecimento para o acúmulo de capacidades que possam conduzir as empresas a um melhor desempenho.

As empresas que tentam implantar ou que sentem a necessidade de um processo relacionado à gestão do conhecimento confundem-se, muitas vezes, por se deparar com uma variedade de processos que respondem sob o nome de gestão do conhecimento. Muitos gestores têm tentado, com sucesso parcial, capturar os ativos do conhecimento centralizando as funções de gestão do conhecimento em um setor específico, como pesquisa e desenvolvimento e/ou recursos humanos, ou investindo em Tecnologia da Informação e Comunicação (TIC). Entretanto, apesar dos esforços, é possível identificar que existem gestores que desconhecem como as empresas podem utilizar o conhecimento em prol de melhores desempenhos. A questão fundamental não é se a empresa tem ou produz conhecimento, mas, como faz seu gerenciamento e aplicação. Nesse sentido, a criação do conhecimento ou transferência pode beneficiar as empresas melhorando suas práticas, inovando e respondendo mais rapidamente ao mercado (LEE; CHOI, 2003; ZHENG; YANG; MCLEAN, 2010).

Em relação à gestão do conhecimento, muitos pesquisadores enfatizam que alguns fatores relacionados à cultura, estrutura, estratégia, a tecnologia de informação e a gestão de pessoas, podem influenciar os processos de gestão do conhecimento (GOLD; MALHOTRA; SEGARS, 2001; LEE; CHOI, 2003; NOH; KIM; JANG, 2014). Segundo Lee e Choi (2003), os fatores facilitadores ou influenciadores da gestão do conhecimento são mecanismos organizacionais que promovem o conhecimento de forma consistente. Noh, Kim e Jang (2014) revelam que os facilitadores de conhecimento direcionam as estruturas organizacionais ao sucesso de uma iniciativa de gestão do conhecimento, desempenhando um papel fundamental no processo de criação e partilha do conhecimento. Dessa forma, os estudos têm se centrado nas análises de fatores relacionados à cultura organizacional como facilitadores da gestão do conhecimento.

Sendo assim, a presente pesquisa tem como objetivo analisar os fatores relacionados à cultura organizacional que auxiliam nos processos de gestão do conhecimento, colaborando para geração de vantagem competitiva nas empresas do setor metalmecânico.

No setor industrial, os valores dos produtos dependem muito mais do percentual de inovação, tecnologia e inteligência a eles incorporadas, do que, propriamente, dos custos de produção. Esses fatores, relacionados ao conhecimento que a empresa possui e utiliza, também conhecidos por ativos intangíveis, são de difícil gerenciamento (SANTIAGO JUNIOR; SPINOLA, 2005), porém, quando tratados de forma eficiente, poderão colocar a empresa em vantagem competitiva.

A inovação tecnológica e a tecnologia de informação e comunicação são características essenciais do setor metalmecânico. Diversos estudos no Brasil vêm relacionando estas características do setor à gestão do conhecimento, tais como Ciconet, Antunes e Ruffoni (2014), Lara e Guimarães (2014), Przyczynski e Vanti (2012) e Tatsch (2008) entre outros. Porém, ainda são raros os estudos que analisam os fatores facilitadores da gestão do conhecimento no setor industrial. Além disso, a maior parte dos estudos realizados no Brasil caracteriza-se como estudos de casos. 
Rápidas mudanças no mercado e na tecnologia estão tornando as estratégias organizacionais cada vez mais difíceis e complexas. Nesse sentido, propõe-se um modelo teórico-empírico de pesquisa, contemplando os elementos facilitadores da gestão do conhecimento, a gestão do conhecimento e os fatores relacionados à vantagem competitiva. Assim, este estudo utiliza-se de um modelo teórico-empírico que possa evidenciar que os fatores culturais contribuem para uma adequada gestão do conhecimento em empresas industriais e que os processos de gestão do conhecimento são estratégicos para a vantagem competitiva das empresas que os possuem.

\section{Referencial Teórico}

Neste capítulo apresenta-se uma seção com os aspectos conceituais relacionados a gestão do conhecimento e seus facilitadores e outra com os aspectos conceituais relacionados a ligação entre a gestão do conhecimento e a vantagem competitiva. Além disso, como última seção apresenta-se o modelo teórico de pesquisa.

\subsection{Gestão do Conhecimento}

Para Nonaka e Von Krogh (2009), o conhecimento é dependente do contexto pessoal e social que um indivíduo está imerso. Desse modo, o conhecimento organizacional está intimamente vinculado ao nível de conhecimento individual. Porém, segundo Nonaka e Von Krogh (2009), a gestão do conhecimento organizacional não se destina apenas a repassar os conhecimentos individuais, mas a complementá-los, tornando-os mais fortes, mais coerentes e amplamente aplicados. Portanto, a gestão do conhecimento representa uma abordagem deliberada e sistemática que garante a utilização total da base de conhecimento da empresa, acoplada com as habilidades individuais, competências, pensamentos, inovações e ideias para criar uma empresa mais eficiente e efetiva.

Embora a literatura acadêmica traga uma variedade de conceitos ligados ao tema conhecimento, conceituá-lo, ainda é uma tarefa complexa. Sendo assim, diversos estudos têm se dedicado a classificá-lo, ao invés de conceituá-lo. Dentre as classificações destacam-se as proposições Karl Polanyi (1966), que mais tarde recebeu importantes contribuições de Nonaka e Takeuchi (1997), dividindo o conhecimento entre tácito e explícito.

Segundo Nonaka e Takeuchi (1997), o conhecimento explícito é o conhecimento que pode ser facilmente expresso em palavras e números, ou seja, pode ser codificado e compartilhado por meio de manuais, documentos e procedimentos padrões de operação. Por outro lado, o conhecimento tácito dificilmente é visível e exprimível, ou seja, é aquele que se adquire pela prática, know how, erros e acertos que estão incorporados nas habilidades dos indivíduos (NONAKA; TAKEUCHI, 1997). Do mesmo modo, pode dizer que está ligado às emoções, valores e ideais dos indivíduos e, por esse motivo, é de difícil transmissão e compartilhamento.

Davenport e Prusak (1998) defendem que a gestão do conhecimento se preocupa com a exploração $e$ o desenvolvimento do conhecimento tácito e explícito de forma que estejam alinhados com os objetivos da organização. Já na percepção de Wong et al. (2013), a gestão do conhecimento pode ser sintetizada como a gestão dos recursos e dos processos de conhecimento da empresa, objetivando através do uso do conhecimento criar valor que resultem em vantagem competitiva.

Nesse sentido, a criação do conhecimento ocorre pela interação entre o conhecimento tácito e explícito, gerando um ciclo criativo, ou seja, uma espiral do conhecimento (NONAKA; TAKEUCHI, 1997). Dessa forma, em seu estudo Nonaka e Takeuchi (1997) propõem quatro processos de interação entre o conhecimento tácito e explicito, os quais são: Socialização, Externalização, Combinação e Internalização. Esses quatro processos são anacronicamente conhecidos por SECI, por meio dos quais, o conhecimento tácito e explicito se difunde em quantidade e qualidade. A passagem ascendente do conhecimento criado em nível individual para o nível grupal, organizacional e interorganizacional caracteriza-se como o espiral do conhecimento.

Para melhor compreensão do modelo SECI, descreve-se os quatro processos de interação entre o conhecimento tácito e explícito (NONAKA; TAKEUCHI, 1997):

a) Socialização - Consiste na partilha do conhecimento tácito. Considera que o co- 
nhecimento é adquirido por meio da criação de modelos mentais, das experiências e do compartilhamento de técnicas, com ou sem o uso da linguagem. Baseia-se nas atividades do dia a dia. Em um contexto organizacional a própria difusão ou divulgação de determinada habilidade técnica implica na socialização do conhecimento.

b) Externalização - Converte o conhecimento tácito em conceitos explícitos. Este processo está fortemente baseado no uso de metáforas $e$ analogias, conceitos e hipóteses. As analogias feitas através do uso de metáforas reduzem o desconhecido, destacando o caráter comum entre dois conceitos diferentes com o intuito de facilitar o entendimento de um deles. Por meio desse processo, as experiências perdem a característica de foro pessoal e podem ser classificadas por ordens gerais de significados, podendo ser reconhecidas pela coletividade.

c) Combinação - Consiste na articulação de novos conhecimentos explícitos a partir do conhecimento explícito existente. O processo envolve a captura e a integração de novos conhecimentos, seguido de sua combinação e disseminação em toda organização por intermédio de reuniões, documentos, conversas ao telefone, e-mails, conferências on-line e apresentações. Como exemplo deste processo, cita-se a criação do conhecimento realizado pela educação e pelo treinamento formal nas escolas.

d) Internalização - Converte o conhecimento explícito em conhecimento tácito. Consiste na ideia de aprender fazendo. As experiências tornam-se ativos valiosos quando internalizadas nas bases do conhecimento tácito dos indivíduos, sob a forma de modelos mentais ou know-how técnico compartilhado. As formas mais comuns de conversão do conhecimento explícito para o tácito são a verbalização $e$ a diagramação do conhecimento sob a forma de documentos, manuais ou histórias orais.

A criação do conhecimento emerge de um processo continuo e dinâmico, envolvendo flutuações entre os quatro modos de conversão propostos por Nonaka e Takeuchi (1997). Esses processos se dão de forma natural no cotidiano das organizações, no entanto, segundo Davenport e Prusak (1998), existem alguns fatores inibidores da transferência de conhecimento que estão relacionados a aspectos culturais, tais como: falta de confiança mútua; diferenças entre culturas e vocabulários; falta de tempo e de locais de encontro; sensação que a recompensa será para os possuidores de conhecimento; falta de capacidade de absorção pelos receptores; a crença de que o conhecimento é privilégio de determinados grupos (síndrome do não inventado aqui); e a intolerância para com os erros ou necessidades de ajuda. Todos esses fatores são condicionadores do sucesso e da eficácia da criação do conhecimento organizacional, pois os valores, normas e comportamentos que constituem a cultura organizacional são os principais determinantes de sucesso.

Lee e Choi (2003) e Noh, Kim e Jang (2014) tratam dos facilitadores da gestão do conhecimento, definindo-os como os fatores que fornecem estruturas essenciais para aumentar a eficiência do processo de conhecimento. Os facilitadores desempenham um papel fundamental no mecanismo de criação, desenvolvimento e partilha do conhecimento organizacional.

Lee e Choi (2003) desenvolveram um modelo de pesquisa considerando os fatores culturais (colaboração, confiança e aprendizagem), de estrutura (centralização e formalização), de pessoas (habilidades) e de tecnologia de informação (suporte de TI) como facilitadores da gestão do conhecimento $e$ influenciadores da performance organizacional. Dos facilitadores analisados, os culturais foram os mais importantes para a gestão do conhecimento. De tal modo, que Allameh, Zare e Davoodi (2011) utilizaram o modelo proposto por Lee e Choi (2003) e identificaram que o suporte $\mathrm{de} \mathrm{TI} e$ a cultura tem o maior efeito sobre os processos de gestão do conhecimento.

Noh, Kim e Jang (2014) investigaram a relação entre a cultura baseada no conhecimento (relacionando os fatores cooperação nas equipes, confiança e aprendizagem organizacional) e o desempenho organizacional, utilizando como variáveis mediadoras a criação $e$ partilha de conhecimento. No entanto, neste estudo apenas a cooperação nas equipes $e$ a aprendizagem organizacional foram significativas para a criação $e$ para a partilha do conhecimento, sendo que a hipótese relacionada à confiança foi rejeitada.

Alvarenga Neto e Choo (2010) verificaram as condições capacitadoras da gestão do conhecimento citadas em 135 artigos da área. Desse modo, constataram que às condições sociocomportamentais, as 
quais envolvem normas e valores e que guiam relações e interações, são capazes de formar um solo fértil para a criação, compartilhamento e uso de conhecimentos, além de facilitar, estimular e recompensar o pensamento inovador. Observaram ainda, que os fatores sociocomportamentais identificados e que podem servir de guia para avaliações de desempenho, contratações, treinamentos, retenções e recompensas foram: confiança mútua; piedade no julgamento (não há perda de status por não se saber tudo); tolerância aos erros honestos; empatia ativa; respeito mútuo; diálogo aberto; ideação e autonomia; entre outros.

Frente aos estudos destacados, pode-se observar que os fatores culturais são repetidamente citados como capazes de moldar a capacidade de uma empresa gerir o seu conhecimento de forma eficaz. Entre os fatores culturais, a colaboração é indicada como um dos fatores capazes de facilitar a troca de conhecimento.

A colaboração pode ser definida como o grau em que as pessoas em um grupo contribuem ativamente para com outros nos seus trabalhos (HURLEY; HULT, 1998). Dessa forma, é possível as diferenças individuais e promove-se a criação do conhecimento (LEE; CHOI, 2003). A cultura de cooperação é o principal meio para que se gerem e se compartilhem as novas informações dentro de equipes (TEECE, 1998). De tal modo, que através da colaboração, os colaboradores podem criar novos conhecimentos por meio da partilha de experiências e informações (BROWN; DUGUID, 1991). Com base nos argumentos apresenta-se a seguinte hipótese de pesquisa:

H1: Existe uma relação positiva entre a colaboração e os processos de criação do conhecimento.

Além da colaboração, alguns autores (LEE; CHOI, 2003; ALVARENGA NETO; CHOO, 2010) assinalam que a confiança também afeta o processo de criação do conhecimento. Segundo Nelson e Cooprider (1996), a confiança é a crença de que a partilha de conhecimentos com os colegas não vai se transformar em uma desvantagem; isto permite a troca de informação entre eles.

Da mesma forma, Lee e Choi (2003) revelam que a confiança afeta o processo de combinação, com isso, percebe-se que simplesmente melhorar a infraestrutura de TI não proporciona uma vantagem competitiva para a combinação do conhecimento. A confiança pode aumentar a vontade dos indivíduos em envolver-se na partilha de conhecimentos, sendo assim, a confiança pode ser considerada uma facilitadora de relações positivas entre os parceiros contratuais (WONG; CHEUNG, 2004). Desse modo, tendo em vista os argumentos apresentados, elaborou-se a seguinte hipótese de pesquisa:

$\mathrm{H} 2$ : Existe uma relação positiva entre a confiança e os processos de criação do conhecimento.

A aprendizagem refere-se ao grau que a aquisição de novos conhecimentos é estimulada nas empresas (HURLEY; HULT, 1998; NOH; KIN; JAN, 2014). Dessa forma, uma cultura voltada para a aprendizagem $e$ desenvolvimento, pode ajudar as pessoas a desempenhar papéis mais ativos na criação de conhecimento nas empresas (LEE; CHOI, 2003). De acordo com o estudo de Noh, Kin e Jang (2014), para que haja uma gestão do conhecimento bem-sucedida é fundamental que a empresa desenvolva métodos de aprendizagem eficazes. Esse estudo mostrou um efeito positivo entre a aprendizagem organizacional $e$ a criação $e$ o compartilhamento de conhecimento. Portanto, as empresas dispostas a aprender constantemente tem maior facilidade em manter sua competitividade e a realizar uma melhor gestão do conhecimento. A partir desses argumentos apresenta-se a seguinte hipótese de pesquisa:

H3: Existe uma relação positiva entre a aprendizagem e os processos de criação do conhecimento.

\subsection{Vantagem Competitiva}

A gestão estratégica tem se concentrado em compreender as fontes de vantagem competitiva das empresas. Desse modo, a literatura de gestão estratégica tem apontado a visão baseada em recursos ou Resource Based View (RBV) como uma das principais perspectivas teóricas. A RBV postula que a vantagem competitiva das empresas provém de uma coleção de recursos que são valiosos, raros, imperfeitamente imitáveis e sustentáveis (BARNEY, 1991).

Por outro lado, os pesquisadores vêm identificando que os recursos da gestão do conhecimento servem como potencial fonte de vantagem competitiva. Desse modo, Grover e Davenport (2001) e Okunoye e Karsten (2002) descrevem a estratégia, a estrutura, a cultura e a tecnologia em relação ao processo de conhecimento 
como as principais fontes de crescimento do negócio e melhoria da competitividade das empresas estudadas.

Outros estudos testam empiricamente a vantagem competitiva a partir de fatores relacionados à gestão do conhecimento. Dentre esses estudos, há os realizados por Gold, Malhotra e Segars (2001), Lee e Choi (2003), Chuang (2004) e Zheng, Yang e McLean (2010) que apontam como indicadores de vantagem competitiva, o desempenho organizacional e a efetividade organizacional.

Para Lee e Choi (2003), o desempenho organizacional pode ser definido como o grau em que as empresas atingem os seus objetivos de negócios. Ele pode ser medido em termos de aprendizagem organizacional, rentabilidade, bem como outros benefícios financeiros da gestão do conhecimento. Essas argumentações sugestionam a seguinte hipótese de pesquisa:

H4: Existe uma relação positiva entre os processos de criação de conhecimento $e$ o desempenho organizacional.

De acordo com o estudo de Zheng, Yang e McLean (2004), a efetividade organizacional é medida pelo grau em que uma empresa realiza seus objetivos. Esse estudo indica ainda, que as medidas de efetividade organizacional - adaptadas de Lee e Choi (2003) - avaliam o grau de percepção dos membros em relação ao sucesso da empresa, a participação de mercado, a rentabilidade, a taxa de crescimento, a capacidade de inovação da empresa em comparação com os principais concorrentes.

Gold, Malhotra e Segars (2001) expõem que semelhante a qualquer recurso organizacional, a gestão do conhecimento eficaz através do desenvolvimento de capacidades deve contribuir para aspectos-chave do desempenho organizacional. Nesse estudo, apresenta-se ainda, uma revisão da literatura sobre gestão estratégica, identificando alguns aspectos-chaves da efetividade organizacional, como: o aperfeiçoamento da capacidade de inovação, o aperfeiçoamento dos esforços de coordenação, a rápida comercialização de novos produtos, a habilidade de antecipar surpresas, a capacidade de resposta às mudanças de mercado e a capacidade de reduzir a redundância de informações e conhecimento. Em consequência desses argumentos, apresenta-se a seguinte hipótese de pesquisa:

H5: Existe uma relação positiva entre os processos de criação de conhecimento e a efetividade organizacional.

\subsection{Modelo Teórico de Pesquisa}

Para o desenvolvimento do estudo elaborou-se um modelo teórico de pesquisa. Esse modelo assume que os fatores de entrada afetam o desempenho de saída através de determinados tipos de processos de interação. Desse modo, os facilitadores de gestão do conhecimento afetam o desempenho e a efetividade organizacional através de processos de criação do conhecimento. Essa relação também é explicada por Lee e Choi (2003) e por Noh, Kim e Jang (2014), porém, esse modelo não utilizar os demais fatores facilitadores propostos pelos estudos anteriores.

Dessa forma, as hipóteses de pesquisa orientam a elaboração de um modelo teórico, com a finalidade de analisar os fatores relacionados à cultura organizacional que auxiliam nos processos de gestão do conhecimento, colaborando para geração de vantagem competitiva nas empresas (Figura 1). As hipóteses de pesquisa apresentadas são provenientes da literatura, permitindo a análise das possíveis relações entre as variáveis de estudo: colaboração, confiança, aprendizagem, criação do conhecimento, desempenho organizacional e efetividade organizacional.

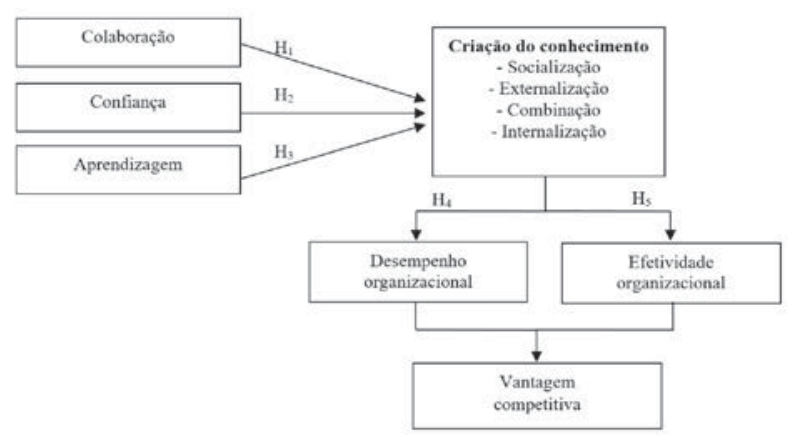

Figura 1: Modelo teórico de pesquisa

Fonte: Adaptada de Lee e Choi (2003)

\section{Método}

Os procedimentos metodológicos do estudo estão baseados na pesquisa quantitativa com estratégia de levantamento de dados. Esse tipo de pesquisa tem como objetivo a descrição das características de determinada população ou de determinado fenômeno, ou então, o estabelecimento de associações entre variáveis (RICHARDSON, 1999).

A população em estudo compreende as empresas do setor metalmecânico da região noroeste do 
Estado do Rio Grande do Sul. Segundo a Federação das Indústrias do Rio Grande do Sul, as categorias de atividade pertencentes ao setor metalmecânico são; metalurgia, produtos de metal, equipamentos de informática e eletrônicos, materiais elétricos, máquinas e equipamentos, veículos automotores, outros equipamentos de transporte, manutenção, reparação e instalação de máquinas e equipamentos (FIERGS, 2014). A amostragem foi realizada por conveniência, ou seja, de acordo com a acessibilidade e a disponibilidade em participar da pesquisa. Com isso, participaram da pesquisa 120 profissionais em 20 empresas do ramo da região noroeste do Estado do Rio Grande do Sul.

Além disso, foi utilizada a classificação do Serviço Brasileiro de Apoio a Micro e Pequenas Empresas para identificação do porte das empresas, que considera: microempresas possuem até 19 funcionários; pequenas possuem de 20 a 99 funcionários; médias empresas possuem de 100 a 500 funcionários; e grandes empresas possuem mais de 500 funcionários (SEBRAE, 2014).

A coleta de dados ocorreu por meio de um questionário, o qual continha os seguintes tópicos: (1) informações sobre a pesquisa; (2) afirmações para a testagem de hipóteses; (3) caracterização da empresa e do respondente. No tópico 2 , para a testagem das hipóteses, as variáveis relacionadas no estudo foram mensuradas com base em uma escala de Likert de onze pontos, variando de (0) "discordo totalmente" a (10) "concordo totalmente".

O questionário foi desenvolvido com base em instrumentos já existentes na literatura de gestão do conhecimento e RBV (Quadro 1). O questionário, primeiramente foi submetido a avaliação de dois especialistas. Após a avaliação dos especialistas, o questionário foi submetido a um pré-teste qualitativo a cinco profissionais de nível médio, gerentes em empresas da mesma região onde a pesquisa foi realizada. Em relação ao pré-teste, as questões que não eram de fácil entendimento para os respondentes foram adequadas e novamente submetidas aos especialistas e após, novamente aos respondentes do pré-teste.

$\mathrm{Na}$ aplicação do questionário considerou-se como respondente os colaboradores de cargos técnico-analistas ou de gestão. Em cada empresa, dois ou mais colaboradores responderam a pesquisa, desse modo, foi possível comparar as respostas, garantindo a fidedignidade na analise de cada empresa.

Dessa forma, para aplicação do questionário foi realizada uma explicação do objetivo da pesquisa por parte dos pesquisadores e após a pesquisa foi entregue a um colaborador da empresa, o qual ficou responsável pela aplicação. Após uma semana, os pesquisadores retornaram às empresas para recolher os questionários. Cabe ressaltar, que visando à fidedignidade da pesquisa, a entrega e o recolhimento da pesquisa foram feitos dentro de envelopes de cor parda, sem nenhuma caracterização, buscando com isso, o anonimato tanto da empresa, quanto dos respondentes.

\begin{tabular}{|c|c|c|}
\hline VARIÁvEIS & DEFINIÇÕES & Estudos \\
\hline Colaboração & $\begin{array}{l}\text { Grau de ajuda e suporte } \\
\text { ativo da organização. }\end{array}$ & $\begin{array}{l}\text { Lee e Choi } \\
\text { (2003) }\end{array}$ \\
\hline Confiança & $\begin{array}{l}\text { Grau de fé recíproca na } \\
\text { intenção, comportamento e } \\
\text { habilidades em relação às } \\
\text { metas organizacionais de todos } \\
\text { os membros da organização. }\end{array}$ & $\begin{array}{l}\text { Lee e Choi } \\
(2003)\end{array}$ \\
\hline Aprendizagem & $\begin{array}{l}\text { Grau de oportunidade, varieda- } \\
\text { de, satisfação e encorajamento } \\
\text { para a aprendizagem e desen- } \\
\text { volvimento organizacional. }\end{array}$ & $\begin{array}{l}\text { Lee e Choi } \\
(2003)\end{array}$ \\
\hline Socialização & $\begin{array}{l}\text { Grau de acumulação de } \\
\text { conhecimento tácito, coleta de } \\
\text { informações externas e internas } \\
\text { a organização e transferência } \\
\text { de conhecimento tácito. }\end{array}$ & $\begin{array}{l}\text { Lee e Choi } \\
\text { (2003) } \\
\text { Nonaka e } \\
\text { Takeuchi } \\
\text { (1997) }\end{array}$ \\
\hline Externalização & $\begin{array}{l}\text { Grau de diálogo criativo, uso } \\
\text { de metáforas e troca de ideias. }\end{array}$ & $\begin{array}{l}\text { Lee e Choi } \\
\text { (2003) } \\
\text { Nonaka e } \\
\text { Takeuchi (1997) }\end{array}$ \\
\hline Combinação & $\begin{array}{l}\text { Grau de aquisição e integra- } \\
\text { ção, síntese e processamento e } \\
\text { disseminação do conhecimento. }\end{array}$ & $\begin{array}{l}\text { Lee e Choi } \\
\text { (2003) } \\
\text { Nonaka e } \\
\text { Takeuchi } \\
\text { (1997) }\end{array}$ \\
\hline Internalização & $\begin{array}{l}\text { Grau de experiências pessoais, } \\
\text { simulação e experimentação. }\end{array}$ & $\begin{array}{l}\text { Lee e Choi } \\
\text { (2003) } \\
\text { Nonaka e } \\
\text { Takeuchi } \\
\text { (1997) }\end{array}$ \\
\hline $\begin{array}{l}\text { Desempenho } \\
\text { organizacional }\end{array}$ & $\begin{array}{l}\text { Grau de sucesso global, parti- } \\
\text { cipação de mercado, taxa de } \\
\text { crescimento da rentabilidade e } \\
\text { inovação em comparação com } \\
\text { os principais concorrentes. }\end{array}$ & $\begin{array}{l}\text { Lee e Choi } \\
\text { (2003) } \\
\text { Chuang } \\
\text { (2004) }\end{array}$ \\
\hline $\begin{array}{l}\text { Efetividade } \\
\text { Organizacional }\end{array}$ & $\begin{array}{l}\text { Grau de capacidade de inovar, } \\
\text { coordenar esforços e a rápida } \\
\text { comercialização de novos pro- } \\
\text { dutos. Habilidade de antecipar } \\
\text { surpresas, responsabilidade } \\
\text { pelas mudanças de mercado, } \\
\text { e de reduzir a redundância de } \\
\text { informações e conhecimento. }\end{array}$ & $\begin{array}{l}\text { Gold, } \\
\text { Malhotra e } \\
\text { Segars (2001) } \\
\text { Zheng, Yang } \\
\text { e McLean } \\
\text { (2004) }\end{array}$ \\
\hline
\end{tabular}

Quadro 1: Operacionalização das variáveis Fonte: Elaborado pelos autores deste artigo 
Após a coleta e tabulação dos dados, as análises estatísticas foram realizadas no programa IBM SPSS Statistic, Version 20. O primeiro procedimento estatístico adotado foi o teste de confiabilidade dos construtos por meio da análise do Alfa de Cronbach. Na sequência, foi realizada a estatística descritiva para todas as variáveis, mediante a utilização da média, desvio padrão, assimetria e curtose. Por último, foi realizada a analise de regressão múltipla para a checagem das hipóteses.

A regressão múltipla é o método de análise apropriado para analisar a relação entre uma única variável dependente/critério e várias variáveis independentes/ preditoras (HAIR JR. et al., 2005). Na pesquisa, as variáveis "critério" são: a criação do conhecimento SECI, a socialização, a externalização, a combinação, a internalização, o desempenho e a efetividade organizacional. Para cada variável critério foi proposto um modelo de regressão individual. Já as variáveis predi- toras são: a colaboração, a confiança, a aprendizagem $e$ a criação do conhecimento - SECI.

\section{Resultados e Discussão}

Neste capítulo apresenta-se a análise dos dados referentes as caraterísticas da amostra e, em sequência, a análise dos dados com os resultados e discussões.

\subsection{Características da Amostra}

Foram distribuídos 120 questionários para 20 empresas, dos quais retornaram 66 questionários respondidos de 13 empresas, ou seja, 55\% dos questionários foram respondidos. Dos 66 questionários respondidos, foram eliminados dois, os quais tinham respostas incompletas e um, que através da análise estatística descritiva foi identificado como outlier. A análise dos dados foi realizada com 63 respondentes.

Tabela 1: Características da amostra

\begin{tabular}{|c|c|c|c|c|c|c|c|}
\hline \multicolumn{8}{|c|}{ (A) NÚMERO DE RESPONDENTES POR DEPARTAMENTO } \\
\hline \multicolumn{8}{|c|}{ PORTE DA EMPRESA/NúMERO DE RESPONDENTES } \\
\hline \multicolumn{2}{|l|}{ Setor } & Média Empresa & \multicolumn{2}{|c|}{ Pequena Empresa } & \multicolumn{2}{|c|}{ Micro Empresa } & Total \\
\hline Administrativo & \multicolumn{2}{|c|}{9} & \multicolumn{2}{|c|}{8} & \multicolumn{2}{|c|}{2} & 19 \\
\hline \multicolumn{2}{|l|}{ Contabilidade } & 3 & \multicolumn{2}{|c|}{-} & \multicolumn{2}{|c|}{1} & 4 \\
\hline Pesquisa e Desenvolvimento & & 8 & \multicolumn{2}{|c|}{-} & \multicolumn{2}{|c|}{1} & 9 \\
\hline \multicolumn{2}{|c|}{ Produção } & 7 & \multicolumn{2}{|c|}{8} & \multicolumn{2}{|c|}{8} & 23 \\
\hline \multicolumn{2}{|l|}{ Qualidade } & 0 & \multicolumn{2}{|c|}{3} & \multicolumn{2}{|c|}{-} & 3 \\
\hline Recursos Humanc & \multicolumn{2}{|c|}{2} & \multicolumn{2}{|c|}{1} & \multicolumn{2}{|c|}{-} & 3 \\
\hline Vendas & \multicolumn{2}{|c|}{2} & \multicolumn{2}{|c|}{-} & \multicolumn{2}{|c|}{-} & 2 \\
\hline Total Geral & \multicolumn{2}{|c|}{31} & \multicolumn{2}{|c|}{20} & \multicolumn{2}{|c|}{12} & 63 \\
\hline \multicolumn{8}{|l|}{ (b) Segmento } \\
\hline Segmento & N. de empresas & $\%$ & npresas & N. de & ondentes & & ondentes \\
\hline Máquinas e equipamentos & 4 & &, 8 & & & &, 8 \\
\hline Estruturas Metálicas & 9 & &, 2 & & & &, 2 \\
\hline Total & 13 & & 00 & & & & 00 \\
\hline
\end{tabular}

(c) Cargo dos respondentes

\begin{tabular}{|c|c|}
\hline (c) CARgo dos ResPondentes & N. RESPONDENTES \\
\hline CARGO & 24 \\
\hline Analista/Técnico & 4 \\
\hline Líder de setor & 12 \\
\hline Coordenador & 9 \\
\hline Supervisor & 6 \\
\hline Gerente & 8 \\
\hline Diretor & 63 \\
\hline Total & 63 \\
\hline
\end{tabular}

Fonte: Dados da pesquisa 


\subsection{Análise de Validade dos Construtos e Análise Descritiva}

A confiabilidade das medidas dos construtos foi avaliada usando o Coeficiente Alpha de Cronbach, cujo nível aceitável de confiabilidade é obtido com valores superiores a 0,70 (HAIR JR. et al., 2005). Todos os construtos avaliados neste estudo obtiveram Alpha de Cronbach maior que 0,8 (Tabela 2).

A variável colaboração foi medida através de cinco itens no questionário, porém, para a obtenção de um coeficiente Alpha de Cronbach aceitável, foi necessário excluir o item 2 do questionário, restando, portanto, quatro itens a ser analisados com a variável colaboração. Para as demais variáveis, foram utilizados todos os itens do questionário.

Tabela 2: Análise descritiva e de confiabilidade das variáveis estudadas

\begin{tabular}{|c|c|c|c|}
\hline VARIável & $\begin{array}{c}\text { N. DE } \\
\text { VARIÁveIS } \\
\text { UTILIzAdAS }\end{array}$ & $\begin{array}{c}\text { AlPHA DE } \\
\text { CRONBACH }\end{array}$ & $\begin{array}{c}\text { MÉdIA } \pm \text { DES- } \\
\text { VIO PADRÃo }\end{array}$ \\
\hline Colaboração & 4 & 0,850 & $6,94 \pm 1,52$ \\
\hline Confiança & 6 & 0,923 & $7,34 \pm 1,39$ \\
\hline Aprendizagem & 5 & 0,866 & $7,49 \pm 1,48$ \\
\hline Socialização & 5 & 0,847 & $7,39 \pm 1,51$ \\
\hline Externalização & 5 & 0,818 & $7,67 \pm 1,23$ \\
\hline Combinação & 5 & 0,89 & $7,74 \pm 1,39$ \\
\hline Internalização & 5 & 0,832 & $7,42 \pm 1,50$ \\
\hline *SECI & 4 & 0,868 & $7,55 \pm 1,19$ \\
\hline Desempenho & 8 & 0,895 & $7,74 \pm 1,25$ \\
\hline Efetividade & 12 & 0,946 & $7,60 \pm 1,40$ \\
\hline
\end{tabular}

*SECI = criação do conhecimento igual à média da média dos resultados encontrados para socialização, externalização, combinação e internalização. Fonte: Dados da pesquisa

\subsection{Análise de Regressão e Teste de Hipóteses}

A Tabela 3 apresenta os modelos de regressão obtidos e os coeficientes de regressão. Todos os modelos de regressão apresentaram-se significativos ao nível de $1 \%$ ( $<<0,01$ ), Tabela 3 . O coeficiente de determinação - R2 indica o percentual (\%) de variação nos resultados da variável dependente que é explicado pelo modelo. Na presente pesquisa, os modelos obtidos respondem de 48,6\% (R2=0,486 modelo de combinação) a 74,5\% ( $\mathrm{R} 2=0,745$ modelo de criação do conhecimento), Tabela 3, a variância dos resultados da variável dependente analisada.

Tabela 3: Modelos de regressão propostos, coeficientes de determinação (R2) e valor de F para os respectivos modelos

(A) ENTRE O PROCESSO DE GESTÃO DO CONHECIMENTO E OS FACILITADORES DA GESTÃO DO CONHECIMENTO

\begin{tabular}{|c|c|c|}
\hline MODELO & $R 2$ & $F$ \\
\hline $\begin{array}{c}\mathrm{SECI}=\alpha+\beta 1 \text { COLABORAÇÃO } \\
+\beta 2 \text { CONFIANÇA }+ \\
\beta 3 \text { APRENDIZAGEM }+\epsilon\end{array}$ & 0,745 & $57,520 * * *$ \\
\hline $\begin{array}{c}\text { SOCIALIZAÇÃO }=\alpha+\beta 1 \\
\text { COLABORAÇÃO }+\beta 2 \text { CONFIANÇA + } \\
\beta 3 \text { APRENDIZAGEM }+\epsilon\end{array}$ & 0,662 & $38,529 * * *$ \\
\hline $\begin{array}{c}\text { EXTERNALIZAÇÃO }=\alpha+\beta 1 \\
\text { COLABORAÇÃO }+\beta 2 \text { CONFIANÇA + } \\
\beta 3 \text { APRENDIZAGEM }+\epsilon\end{array}$ & 0,495 & $19,303 * * *$ \\
\hline $\begin{array}{c}\text { COMBINAÇÃO }=\alpha+\beta 1 \text { COLABORAÇÃO } \\
+\beta 2 \text { CONFIANÇA }+ \\
\beta 3 \text { APRENDIZAGEM }+\epsilon\end{array}$ & 0,486 & $18,611^{* * * *}$ \\
\hline $\begin{array}{c}\text { INTERNALIZAÇÃO }=\alpha+\beta 1 \\
\text { COLABORAÇÃO + } \beta 2 \text { CONFIANÇA + } \\
\beta 3 \text { APRENDIZAGEM }+\epsilon\end{array}$ & 0,551 & $24,106 * * *$ \\
\hline
\end{tabular}

(B) ENTRE O PROCESSO DE CRIAÇÃO DO CONHECI-

MENTO E VANTAGEM COMPETITIVA SUSTENTÁVEL

\begin{tabular}{cccc|}
\hline MODELO & $R 2$ & $F$ \\
\hline DESEMPENHO $=\alpha+\beta 1$ SECI $+\epsilon$ & 0,509 & $63,226 * * *$ \\
\hline EFETIVIDADE $=\alpha+\beta 1$ SECI $+\epsilon$ & 0,539 & $71,197 * * *$ \\
\hline
\end{tabular}

*** valor de $\mathrm{p}<0,01$ ou $1 \%$.

Fonte: Dados da pesquisa

A Tabela 4 apresenta um sumário da análise de regressão. A confiança e a aprendizagem são preditores positivos e significativos ( $p<0,05$ e $p<0,01$, Tabela 4) da criação de conhecimento - SECI, sendo que a aprendizagem $(\beta=0,600)$ tem um efeito maior sobre a criação do conhecimento do que a confiança $(\beta=0,195)$, Tabela 2. Portanto, as hipóteses $\mathrm{H} 2$ e H3 foram confirmadas.

A colaboração não apresentou significância ( $p>0,05$, Tabela 4) no modelo de regressão de criação do conhecimento - SECI, portanto a hipótese $\mathrm{H} 1$ deve ser rejeitada.

Além disso, verificou-se também que a criação do conhecimento afeta positiva e significativamente 0 desempenho e efetividade organizacional, confirmando, portanto as hipóteses $\mathrm{H} 4$ e $\mathrm{H} 5$. 
Tabela 4: Sumário dos resultados da análise de regressão

\begin{tabular}{|c|c|c|c|c|c|c|c|c|}
\hline \multicolumn{9}{|c|}{ VARIÁveis DePENDENTES } \\
\hline $\begin{array}{c}\text { VARIÁVEIS } \\
\text { INDEPENDENTES }\end{array}$ & & Socialização & Externalização & Combinação & Internalização & SECI & Desempenho & Efetividade \\
\hline \multirow{2}{*}{ Constante } & $\alpha$ & 0,328 & 3,267 & 1,827 & 0,817 & 1,609 & 2,083 & 1,084 \\
\hline & $\mathrm{t}$ & 0,458 & $4,587 * * *$ & $2,255^{* *}$ & 1,241 & $3,278 * * *$ & $2,894^{* * *}$ & 1,387 \\
\hline \multirow{2}{*}{ Colaboração } & $\beta$ & 0,032 & 0,019 & 0,038 & 0,146 & 0,003 & \multirow{2}{*}{$\mathrm{N} / \mathrm{A}$} & \multirow{2}{*}{$\mathrm{N} / \mathrm{A}$} \\
\hline & $\mathrm{t}$ & 0,248 & 0,146 & 0,262 & $-0,523$ & 0,034 & & \\
\hline \multirow{2}{*}{ Confiança } & $\beta$ & 0,197 & $-0,012$ & 0,312 & 0,158 & 0,195 & \multirow{2}{*}{$\mathrm{N} / \mathrm{A}$} & \multirow{2}{*}{$\mathrm{N} / \mathrm{A}$} \\
\hline & $t$ & 1,426 & $-0,088$ & $1,992 *$ & $1,784 *$ & $2,052^{* *}$ & & \\
\hline \multirow{2}{*}{ Aprendizagem } & $\beta$ & 0,720 & 0,582 & 0,449 & 0,098 & 0,600 & \multirow{2}{*}{$\mathrm{N} / \mathrm{A}$} & \multirow{2}{*}{$\mathrm{N} / \mathrm{A}$} \\
\hline & $\mathrm{t}$ & $8,387^{* * * *}$ & $6,812 * * *$ & $4,618 * * *$ & $6,640 * * *$ & $10,197 * * *$ & & \\
\hline \multirow{2}{*}{ SECI } & $\beta$ & \multirow{2}{*}{$\mathrm{N} / \mathrm{A}$} & \multirow{2}{*}{$\mathrm{N} / \mathrm{A}$} & \multirow{2}{*}{$\mathrm{N} / \mathrm{A}$} & \multirow{2}{*}{$\mathrm{N} / \mathrm{A}$} & \multirow{2}{*}{$\mathrm{N} / \mathrm{A}$} & 0,749 & 0,863 \\
\hline & $t$ & & & & & & $7,951 * * *$ & $8,438 * * *$ \\
\hline
\end{tabular}

Valores negritados apresentam significância estatística. ${ }^{* * *} \mathrm{p}<0,01 ; * * \mathrm{p}<0,05 ; * \mathrm{p}<0,1$. N/A: Não analisado Fonte: Dados da pesquisa

\subsection{Avaliação das Hipóteses}

A hipótese $H 1$, de que existe uma relação positiva entre a colaboração e os processos de criação do conhecimento, não foi confirmada. Isso demonstra que as empresas do setor metalmecânico não apresentaram como relevante para geração do conhecimento o processo de colaboração. Isso contraria os estudos de Lee e Choi (2003) e Noh, Kim e Jang (2014), os quais descrevem que havendo uma cultura colaborativa entre os membros de uma organização, haveria também a criação de conhecimento. Para os autores, a colaboração diminui as diferenças individuais e promove a criação do conhecimento. Entretanto, neste estudo, essa hipótese não foi comprovada. Por quê?

A hipótese $\mathrm{H} 2$ de que existe uma relação positiva entre a confiança e os processos de criação do conhecimento, ficou comprovada. Dessa forma, a confiança é um fator determinante para o processo de criação do conhecimento SECI no setor metalmecânico. A confiança incentiva um clima propício para o trabalho, ou seja, onde existe confiança, existe segurança e entusiasmo em se aprender coisas novas e compartilhar, não só o aprendizado novo, como também as experiências adquiridas ao longo do tempo. Conforme Nahapiet $e$ Ghoshal (1998), a confiança pode ser definida como a manutenção de fé recíproca na intenção e no comportamento dos outros membros da organização.
Além disso, este estudo comprova também que a confiança é um preditor positivo e significativo da combinação e da internalização, ou seja, a confiança estimula a criação de novos conhecimentos explícitos a partir do conhecimento explícito existente e também ajuda na conversão do conhecimento explícito em conhecimento tácito.

Os resultados corroboram com os estudos de Lee e Choi (2003) e Alvarenga Neto e Choo (2010) revelando que quando os relacionamentos são ricos em confiança, as pessoas estão mais dispostas à troca de conhecimento. No entanto, contraria o estudo de Noh, Kim e Jang (2014) em que a confiança não foi um preditor da criação e partilha do conhecimento.

Por meio da confirmação da hipótese $\mathrm{H} 3$, de que existe uma relação positiva entre o processo de aprendizagem e os processos de gestão do conhecimento, a pesquisa demonstra que no setor metalmecânico a organização que investe e estimula o processo de aprendizagem terá melhores resultados na gestão do conhecimento. Quanto maior o estímulo para aquisição de novos conhecimentos e para a aprendizagem, melhor é o desempenho e a disposição em se aplicar e disseminar esse conhecimento na empresa. Nesse sentido, Kanevsky e Housel (1998) verificaram que a quantidade de tempo gasto na aprendizagem está positivamente relacionada com a quantidade de 
conhecimento. Este estudo está de acordo com os resultados anteriores de Lee e Choi (2003), Allameh, Zare e Davoodi (2011) e Noh, Kim e Jang (2014) de que a aprendizagem influencia diretamente todos os processos de gestão do conhecimento.

Além disso, o estudo comprova que a criação do conhecimento afeta significativamente o desempenho da organização no setor metalmecânico, através da aceitação da hipótese $\mathrm{H} 4$ de que existe uma relação positiva entre os processos de criação de conhecimento e o desempenho organizacional. O coeficiente de determinação (R2) para o modelo que relaciona o desempenho organizacional ao processo de criação do conhecimento de Nonaka e Takeuchi foi de 0,509 (Tabela 3), ou seja, aproximadamente $51 \%$ da variação dos resultados de desempenho são explicados pelo modelo. Para Gold, Malhotra e Segars (2001), as empresas com elevada capacidade de gestão do conhecimento em uma área-chave são capazes de responder rapidamente aos movimentos estratégicos pelos concorrentes, o que denota o seu grau de vantagem competitiva. Sendo assim, quanto maior o grau de transferência e compartilhamento de conhecimento dos seus colaboradores, maior é a sua vantagem competitiva.

A hipótese H5 de que existe uma relação positiva entre os processos de criação de conhecimento e a efetividade organizacional, também foi confirmada. Dessa forma, revelando que a criação do conhecimento influencia positivamente e significativamente a efetividade organizacional. A efetividade organizacional está relacionada ao desempenho global de uma empresa na medida em que mede o efeito líquido dos processos de negócios, business processes, sobre a posição da empresa no mercado (RAY; BARNEY; MUHANNA, 2004). Para os autores, a efetividade está relacionada, segundo a visão baseada em recursos, à vantagem competitiva sustentável, pois proporciona a avaliação de quanto os recursos e capacidades das empresas atingem o potencial competitivo delas por meio de seus processos de negócios. Cabe ressaltar, que o estudo demonstrou uma regressão de 53,9\% (R2 $=0,539$, Tabela 3) para o modelo que relaciona a efetividade à gestão do conhecimento, ou seja, pode-se inferir que a gestão do conhecimento tem um importante papel nos processos de negócios e na posição de mercado das empresas do setor metalmecânico, podendo levar a vantagem competitiva das empresas que a implementam eficazmente.
Os resultados da pesquisa em relação à efetividade e ao desempenho organizacional confirmam os estudos anteriores de Gold, Malhotra e Segars (2001), Lee e Choi (2003), Chuang (2004), Zheng, Yang e McLean (2010) e Noh, Kim e Jang (2014) e, dessa forma, consolidam a importância da gestão do conhecimento para a vantagem competitiva das empresas.

\section{Conclusão}

O estudo analisou os fatores relacionados à cultura organizacional que auxiliam nos processos de gestão do conhecimento, colaborando para geração de vantagem competitiva nas empresas do setor metalmecânico. Assim, por meio da análise da regressão múltipla pode-se verificar que aprendizagem e a confiança são preditores positivos e significativos da gestão do conhecimento.

Dessa forma, observa-se a importância do investimento por parte das organizações em esforços para a promoção da cultura organizacional voltada para a busca de oportunidade, variedade, satisfação e encorajamento para a aprendizagem e desenvolvimento do conhecimento organizacional. Além disso, a promoção de uma cultura voltada a manutenção da confiança e da fé recíproca na intenção, comportamento de todos os membros da organização, pode levar as organizações a obtenção de resultados melhores em relação à gestão do conhecimento.

Os resultados deste estudo indicam, ainda, que a gestão do conhecimento influencia o desempenho e a efetividade organizacional. As empresas que têm um ambiente favorável para a criação, uso e compartilhamento do conhecimento tácito e explícito terão mais sucesso na obtenção de vantagem competitiva frente aos concorrentes.

Nesse sentido, a contribuição acadêmica da pesquisa está em evidenciar através de um modelo teórico-empírico que uma cultura de confiança e voltada para a aprendizagem podem contribuir para os processos de gestão do conhecimento, e, por sua vez, para a vantagem competitiva das empresas que os possuem. Assim, o modelo aponta que as empresas que utilizam seus recursos e capacidades gerados pela gestão do conhecimento poderão obter resultados efetivos em seus processos de negócios. 
No entanto, o estudo contrariou os resultados de Lee e Choi (2003) e Noh, Kim e Jang (2014) que haviam encontrado grande significância frente à variável colaboração no sentido de adquirir conhecimento em empresas coreanas. $\mathrm{O}$ estudo voltado estritamente ao setor metalmecânico brasileiro não obteve os mesmos resultados. Sendo que a hipótese demonstrando relação entre colaboração e criação de conhecimento foi descartada. Essa contradição abre espaço para novas investigações, visando desmistificar com ênfase significativa porque no setor metalmecânico a colaboração não é um influenciador para a gestão do conhecimento.

Dessa forma, é possível sugerir, como pesquisa futura, estudos que busquem compreender porque a colaboração em algumas culturas influencia a aquisição e compartilhamento de conhecimento e em outras não. Além disso, sugerem-se pesquisas em profundidade que busquem compreender como os facilitadores culturais da gestão do conhecimento interagem e podem melhor auxiliar outros processo da empresa.

Na realização do estudo algumas dificuldades foram encontradas, entre elas está a falta de compreensão do processo de pesquisa cientifica por parte de algumas empresas. As empresas em prol do sigilo a respeito de suas atividades e práticas sentem-se invadidas quando sugeridas a participar de um processo de pesquisa, especialmente, no tocante a gestão de pessoas. Além disso, os respondentes possuem limitação de tempo em responder aos questionários, uma vez que, concentram sob suas responsabilidades uma série de tarefas cotidianas.

Outra dificuldade encontrada, é que no Brasil não há um distrito industrial organizado por ramo de atividade, diferente, por exemplo, de países europeus, como a Itália que concentra seus segmentos empresariais de acordo com a atividade exercida (BECATTINI, 1999). Sendo assim, as empresas do setor metalmecânico nos dois municípios pesquisados apresentaram distâncias relevantes de localização, dificultando o deslocamento dos pesquisadores.

Portanto, os resultados do presente estudo devem ser analisados a luz de suas limitações inerentes. Primeiramente, este estudo é uma pesquisa de corte transversal, ou seja, um retrato do instante. Resultados mais robustos poderiam ser obtidos através de um estudo longitudinal para avaliar as características dinâmicas da gestão do conhecimento. Além disso, o estudo abrange apenas empresas de micro a médio porte, não contemplando as empresas de grande porte $e$, portanto, mais complexas. Finalmente, os resultados são limitados a empresas do setor metalmecânico da região noroeste do Estado do RS. A generalização para outras regiões ou estados brasileiros pode ser questionável.

\section{Agradecimentos}

Os autores agradecem aos valiosos comentários de Kenny Basso e Marlon Dalmoro, nas versões anteriores, e à CAPES pelo auxílio concedido por meio de uma bolsa PROSUP.

\section{REFERÊNCIAS}

ALLAMEH, S. M.; ZARE, S. M.; DAVOODI, S. M. R. Examining the impact of $\mathrm{KM}$ enablers on knowledge management processes. Procedia Computer Science, [S.l.], v. 3, p. 1.211-1.223, 2011.

ALVARENGA NETO, R. C. D.; CHOO, C. W. Beyond the ba: managing enabling contexts in knowledge organizations. Journal of Knowledge Management, [S.l.], v. 14, n. 4, p. 592-610, 2010.

BARNEY, J. B. Firm resources and sustained competitive advantage. Journal of Management, [S.I.], v. 17, n. 1, p. 99-120, 1991.

BECATTINI, G. Distritos industriais na Itália. In: COCCO, G.; URANI, A.; GALVÃO, A. P. (Org.). Empresários e empregos nos novos territórios produtivos: o caso da terceira Itália. Rio de Janeiro: DP\&A, 1999. p. 45-57.

BROWN, J. S.; DUGUID, P. Organizational learning and communities-of-practice: Toward a unified view of working, learning, and innovation. Organization Science, [S.1.], v. 2, n. 1, p. 40-57, 1991.

CHUANG, S. H. A resource-based perspective on knowledge management capability and competitive advantage: an empirical investigation. Expert Systems with Applications, [S.I.], v. 27, n. 3, p. 459-465, 2004. 
CICONET, I.; ANTUNES JR., J. A. V.; RUFFONI, J. Características das atividades para a geração da inovação no arranjo produtivo local metal mecânico automotivo da região de Caxias do Sul (RS). Revista Brasileira de Gestão e Inovação, [S.l.], v. 1, n. 3, p. 1-26, 2014.

DAVENPORT, T. H.; PRUSAK, L. Working Knowledge: How Organizations Manage What They Know. Boston: Harvard Business School Press, 1998.

DIERICKX, I.; COOL, K. Asset stock accumulation and sustainability of competitive advantage. Management Science, [S.l.], v. 35, n. 12, p. 1.504-1.511, 1989.

FIERGS. Federação das Indústrias do Rio Grande do Sul. Caderno Setorial Rio Grande do Sul: Setor metal mecânico. Unidade de Estudos Econômicos - UEE, 2011. Disponível em: <http://adesm.org.br/wp-content/ uploads/2011/11/Metal-Mec\%C3\%A2nico-FIERGS.pdf> . Acesso em: 30 nov. 2014.

GOLD, A. H.; MALHOTRA, A.; SEGARS, A. H. Knowledge management: an organizational capabilities perspective. Journal of Management Information Systems, [S.l.], v. 18, n. 1, p. 185-214, 2001.

GRANT, R. M. The resource based theory of competitive advantage: implications for strategy formulation.

California Management Review, [S.l.], v. 33, n. 3, p. 114-135, 1991.

GROVER, V.; DAVENPORT, T. H. General perspectives on knowledge management: Fostering a research agenda. Journal of Management Information Systems, [S.I.], v. 18, n.1, p. 5-21, 2001.

HAIR JR., J. F. et al. Fundamentos de métodos de pesquisa em administração. Porto Alegre: Bookmam, 2005.

HURLEY, R.; HULT. T. Innovation, market orientation, and organizational leaming: An integration and empirical examination. Journal of Marketing, [S.l.], v. 62, n. 3, p. 45-54, 1998.

KANEVSKY; V., HOUSEL; T. The learning-knowledgevalue cycle. In: VON KROGH; G., ROOS; J., KLEINE; D. (Org.). Knowing in Firms: Understanding, Managing and Measuring Knowledge. London: Sage, 1998. p. 269-284.
LARA, F. F; GUIMARÃES, M. R. N. Competitive priorities and innovation in SMEs: A Brazil multi-case study.

Journal of Technology Management \& Innovation, [S.l.], v. 9, n. 3, p. 51-64, 2014.

LEE, H.; CHOI, B. Knowledge management enablers, processes, and organizational performance: an integrative view and empirical examination. Journal of Management Information Systems, [S.I.], v. 20, n. 1, p. 179-228, 2003.

NAHAPIET, J.; GHOSHAL, S. Social capital, intellectual capital, and the organizational advantage. Academy of Management Review, [S.1.], v. 23, n. 2, p. 242-266, 1998.

NELSON, K. M.; COOPRIDER, J. G. The contribution of shared knowledge to IS group performance. MIS Quarterly, [S.l.], v. 20, n. 4, p. 409-432, 1996.

NOH, M.; KIM, H.; JANG, H. Learning performance and business performance of knowledge management organizations: the moderating effect of technological capability. Information Development, [S.I.], p. 1-18, 2014.

NONAKA, I.; TAKEUCHI, H. Criação de conhecimento na empresa: como as empresas japonesas geram a dinâmica da inovação. 14. ed. Rio de Janeiro: Elsevier, 1997.

NONAKA, I.; VON KROGH, G. Perspective-tacit knowledge and knowledge conversion: Controversy and advancement in organizational knowledge creation theory. Organization Science, [S.1.], v. 20, n. 3, p. 635-652, 2009.

OKUNOYE, A.; KARSTEN, H. Where the global needs the local: variation in enablers in the knowledge management process. Journal of Global Information Technology

Management, [S.I.], v. 5, n. 3, p. 12-31, 2002.

POLANYI, M. The tacit dimension. New York: Doubleday and Co., 1966.

PORTER, M. E. Estratégia competitiva: técnicas para análise de indústrias e da concorrência. Rio de Janeiro: Campus, 1986.

PRAHALAD, C.; HAMEL, G. The core competence of the corporation. Harvad Business Review, [S.I.], v. 68, n. 3, p. 79-91, 1990. 
PRZYCZYNSKI, R.; VANTI, A. A. Recursos de tecnologia da informação sustentadores de vantagem competitiva: um estudo no setor metal mecânico agroindustrial.

Revista de Administração Mackenzie, [S.l.], v. 13, n. 4, p. 171-205, 2012.

RAY, G.; BARNEY, J. B.; MUHANNA, W. A. Capabilities, business processes, and competitive advantage: choosing the dependent variable in empirical tests of the resourcebased view. Strategic Management Journal, [S.I.], v. 25, n. 1, p. 23-37, 2004.

RICHARDSON, R. J. Pesquisa social: métodos e técnicas. 3. ed. São Paulo: Atlas, 1999.

SANTIAGO JUNIOR, J. R. S.; SPINOLA, M. M. A gestão do conhecimento na empresa: um estudo de caso na construção civil. In: NETO, J. A. (Org.) Redes entre organizações: domínio do conhecimento e da eficácia operacional. São Paulo: Atlas, 2005. p. 147-169.

SEBRAE - Serviço Brasileiro de Apoio a Micro e Pequenas Empresas. Critérios de classificação de empresas. [2014]. Disponível em: <http://www.sebraesc.com.br/leis/default.asp?vcdtexto $=4154>$. Acesso em: 15 dez. 2014.

TATSCH, A. L. Conhecimento, aprendizagem, inovação e proximidade espacial: o caso do arranjo de máquinas e implementos agrícolas no Rio Grande do Sul. Revista Brasileira de Inovação, [S.1.], v. 7, n. 1, p. 63-100, 2008.

TEECE, D. J. Capturing value from knowledge assets: The new economy, markets for know-how and intangible assets. California Management Review, [S.l.], v. 40, n. 3, p. 55-79, 1998.

WERNERFELT, B. A resource-based view of the firm.

Strategic Management Journal, [S.l.], v. 5, n. 2, p. 171180, 1984.

WONG, K. Y. et al. Knowledge management performance measurement: measures, approaches, trends and future directions. Information Development, [S.l.], p. 1-19, 2013.

WONG, P. S.; CHEUNG, S. Trust in construction partnering: views from parties of the partnering dance. International Journal of Project Management,
[S.l.], v. 22, n. 6, p. 437-446, 2004.

ZANDER, U; KOGUT, B. Knowledge and the speed of the transfer and imitation of organizational capabilities: an empirical test. Organizational Science, [S.I.], v. 6, n. 1, p. 76-92, 1995.

ZHENG, W.; YANG, B.; MCLEAN, G. N. Linking organizational culture, structure, strategy, and organizational effectiveness: mediating role of knowledge management. Journal of Business Research, [S.I.], v. 63, n. 7, p. 763-771, 2010. 